\title{
Inertial Automorphisms of an Abelian Group
}

\author{
Ulderico Dardano (*) - Silvana Rinauro (**)
}

\begin{abstract}
An automorphisms $\gamma$ of a group is inertial if $X \cap X^{\gamma}$ has finite index in both $X$ and $X^{\gamma}$ for any subgroup $X$. We study inertial automorphisms of abelian groups and give characterization of them. In particular, if the group is periodic they have property that $X^{\langle\gamma\rangle} / X_{\langle\gamma\rangle}$ is bounded. We also study finitely generated groups of inertial automorphisms.
\end{abstract}

\section{Introduction}

Recently there has been some interest in groups whose subgroups are inert, that is commensurable to their conjugates (see [1], [7]). Here two subgroups $X$ and $Y$ of a group are told commensurable iff the index of $X \cap Y$ in both $X$ and $Y$ is finite. Commensurability is an equivalence relation.

The study of the above groups yields to the consideration of inertial automorphisms, that is automorphisms mapping subgroups to commensurable subgroups. In this paper we give characterization of these automorphisms of an abelian group $A$. In Theorem 1 we show that when $A$ is torsion they reduce to so-called almost power automorphisms, that is automorphisms with property ( $a p$ ) below, which have been studied in [3] by Franciosi-de Giovanni-Newell. Further, we show that even in the nonperiodic case almost power automorphisms coincide with automorphisms with the property $(b p)$, which have been studied in [2] by Casolo.

(*) Indirizzo dell'A.: Dipartimento di Matematica e Applicazioni "R.Caccioppoli”, Università di Napoli “Federico II”, Via Cintia - Monte S. Angelo, I-80126 Napoli,

E-mail:dardano@unina.it

(**) Indirizzo dell'A.: Dipartimento di Matematica e Informatica, Università della Basilicata, Via dell'Ateneo Lucano 10 - Contrada Macchia Romana, I-85100 Potenza, Italy.

E-mail: silvana.rinauro@unibas.it 
More precisely, we consider following properties for an automorphism $\gamma$ of an abelian group $A$. If $\Gamma$ is a group acting on a group $G$ and $X \leq G$, as usual we denote $X_{\Gamma}:=\cap_{\gamma \in \Gamma} X^{\gamma}$ and $X^{\Gamma}:=\left\langle X^{\gamma} \mid \gamma \in \Gamma\right\rangle$.

(ap) $\forall X \leq A \quad\left|X / X_{\langle\gamma\rangle}\right|<\infty \quad$ ( $\gamma$ is almost power);

(bp) $\forall X \leq A \quad\left|X^{\langle\gamma\rangle} / X\right|<\infty ;$

(in) $\forall X \leq A \quad\left|X / X \cap X^{\gamma}\right| \cdot\left|X^{\gamma} / X \cap X^{\gamma}\right|<\infty$ ( $\gamma$ is inertial);

(bin) $\exists k \in \mathbb{N} \quad \forall X \leq A \quad\left|X / X \cap X^{\gamma}\right| \cdot\left|X^{\gamma} / X \cap X^{\gamma}\right| \leq k$.

By Theorem 1 we see that in the periodic case they correspond to the fact that $\gamma$ acts on a finite index subgroup (or modulo a finite subgroup) either as a power (elementary case) or pseudo-power automorphism (see Definition 1). Furthermore the above properties are equivalent to each of the following:

$\left(d_{*}\right)$ there exists $d_{*} \in \mathbb{N}$ such that $\left|X / X_{\langle\gamma\rangle}\right| \leq d_{*}$ for all $X \leq A$.

(d) there exists $d \in \mathbb{N}$ such that $\left|X^{\langle\gamma\rangle} / X\right| \leq d$ for all $X \leq A$.

Thus such a $\gamma$ is inertial in a "bounded" way. Moreover, all the relevant indeces are bounded each in terms of any other. We give also several examples to show that many given bounds are best-possible.

Theorem 2 identifies inertial automorphisms of torsion-free abelian groups, while Theorem 3 treats the mixed case. It follows a characterization of "bounded" inertial automorphisms.

In the final section we apply the above results to finitely generated groups of inertial automorphisms.

\section{Notation and Terminology}

We use multiplicative notation for abelian group operation, except in Section 3. Most of our notation is standard and refers to [4] and [6]. In particular $D(A), T(A), \exp (A), \operatorname{Aut}(A)$ denote the maximum divisible subgroup, the torsion subgroup, the exponent, the automorphism group of the abelian group $A$, resp. Also, if $n \in Z, A[n]$ is the subgroup of the elements such that $x^{n}=1$ and $\pi(n)$ denote the set of prime divisors of $n$. If $\pi$ a set of primes we denote by $A_{\pi}$ the $\pi$-component of $A$ while by $Q_{\pi}$ we denote the group of rationals whose denominators can be written by a product of primes in $\pi$ only.

Recall that a power automorphism is an automorphism mapping each subgroups into itself. Power automorphisms of an abelian non-periodic group are just the identity or the inversion map. Futher, power automorphisms of an abelian $p$-group are universal, that is have form $x \mapsto x^{\alpha}$, where $\alpha$ is an invertible $p$-adic number. 
If $\gamma \in \operatorname{Aut}(G)$ and $C \leq B \leq A$ are such that $\gamma$ induces a power automorphism corresponding to the $p$-adic $\alpha$ on the $p$-group $B / C$ we write $\gamma_{\mid B / C}=\alpha$. Clearly, $B^{\gamma-\alpha} \leq C$.

\section{Periodic case}

Let us start recalling that when dealing with torsion groups we can reduce to $p$-groups.

Proposition 1. Let $G=D r_{i} G_{i}$ be the direct product of coprime torsion groups and $\gamma \in \operatorname{Aut}(A)$. Then $\gamma$ is either (ap), (bp), (in) or (bin) iff it acts this way on finitely many $G_{i}$ 's and as a power on all the others.

Let us point out an easy fact which involves some inertial automorphism that we can call elementary.

Proposition 2. Let $A$ be an abelian group and $\gamma \in \operatorname{Aut}(A)$. Then:

i) If $\gamma$ is power on a finite index subgroup $B$, then $\gamma$ is power on a factor $A / C$ with $|C| \leq|A / B|$.

ii) If $\gamma$ is power on a factor $A / C$ with $C$ finite, then $\gamma$ is power on a subgroup $B$ with $|A / B| \leq|C|$.

Proof. If $A$ is periodic, we can assume $A$ is a $p$-group and consider the $p$-adic $\alpha$ such that $\gamma=\alpha$ on $B$ or on $A / C$, accordingly we are in the hypotheses of statement $(i)$ or $(i i)$. For the non-periodic case in the same way let $\alpha= \pm 1$. Now denote by $K$ and $I$ the kernel and the image of the homomorphism $\gamma-\alpha$, respectively. Then $\gamma=\alpha$ on $B \leq A$ iff $B \leq K$ and $\gamma=\alpha$ on $A / C$ if and only if $I \leq C$. Clearly $A / K \simeq_{\gamma} I$.

Let us give a definition which will simplify statements and whose idea is in [2] and [3] already. It deals with non-elementary almost power automorphism, as we see in Proposition 3.

Definition 1. Let $p$ be a prime, $r, \varepsilon \in \mathbb{N}$ and $e=p^{\varepsilon}$. An abelian $p$ group $A$ is told $(r, e)$-bounded iff the largest divisible subgroup $D=D(A)$ of $A$ has rank $\leq r$ and $A / D$ has exponent dividing $e$.

If $\gamma \in \operatorname{Aut}(A)$ we say that $\gamma$ a pseudo-power automorphism of $A$ if it is non-power and $A=D \times E$ where: 

i) $D$ is divisible with finite rank $r$,
ii) $E$ is infinite but has finite exponent $e$,
iii) $\gamma$ acts as a power automorphism on both $D$ and $E$.

Proposition 3. Let $p$ be a prime, $r, \varepsilon \in \mathbb{N}$ and $e=p^{\varepsilon}$.

(i) If $\gamma$ is a pseudo-power automorphism of an abelian $(r, e)$-bounded p-group, then for each $X \leq A$ we have

$$
\left|X^{\langle\gamma\rangle} / X\right| \leq e^{r} \text { and }\left|X / X_{\langle\gamma\rangle}\right| \leq e^{r} .
$$

(ii) Ifp $>2$, there exists a pseudo-power automorphism $\gamma$ of an abelian $(r, e)$-bounded p-group $A$ with a subgroup $X \leq A$ such that

$$
\left|X^{\langle\gamma\rangle} / X\right|=\left|X / X_{\langle\gamma\rangle}\right|=e^{r} .
$$

Proof. i) In the same notation as in Definition 1, if $X^{*}:=D X \cap X E$, $X_{*}:=(D \cap X)(X \cap E)$ we have that $X_{*}$ and $X^{*}$ are $\gamma$-invariant while the groups $X^{*} / X \simeq X / X_{*} \simeq(A \cap X B) /(A \cap X) \simeq(A X \cap B) /(X \cap B)$ have rank $\leq r$ and exponent $\leq e$.

ii) Let $A_{1}:=D \times V$, where $D$ is a Prüfer $p$-group and $V=\langle v\rangle$ has order $p^{\varepsilon}$. Define $\gamma$ by $\gamma_{\mid V}=1$ and $\gamma_{\mid D}=\alpha \neq \equiv 1(\bmod p)$. Let $X_{1}:=\langle v d\rangle$, where $d \in D$ has order $p^{\varepsilon}$. Write $y=v^{m} d^{m}=v^{n} d^{\alpha n} \in X_{1} \cap X_{1}^{\gamma}$, with $0 \leq m, n<p^{\varepsilon}$. Then $n \equiv m$ and $n \equiv \operatorname{m\alpha }\left(\bmod p^{\varepsilon}\right)$. Hence $m(\alpha-1) \equiv 0$ $\left(\bmod p^{\varepsilon}\right)$, so that $v^{m}=1$ and $X_{1} \cap X_{1}^{\gamma}=1$.

Let now $A$ be the direct product of $r$ copies of $A_{1}$ by an infinite abelian group $Y$ of exponent $e$ and define $\gamma$ on each copy of $A_{1}$ as above and by $\gamma_{\mid Y}=1$. Then $\gamma$ is pseudo-power on $A$ and the statement holds when $X$ is the subgroup generated by the $r$ subgroups corresponding to $X_{1}$.

Notice that, even tough it is plain that $A$ splits on $D=D(A)$, the fact that $\gamma$ acts as a power on both $D$ and $A / D$ (with finite rank and exponent, resp.) is not enough to guarantee that $\gamma$ is either power or pseudo-power on $A$. To see this consider a group $A=D \times\langle b\rangle$ where $D$ is a Prüfer $p$-group and $b$ has order $p$ with $1 \neq \gamma_{\mid D}=\alpha \equiv 1(\bmod p)$ and $b^{\gamma}=a b$, where $a \in D$ has order $p$. Clearly $\gamma$ acts as a power on both $D$ and $A / D$. However, if $A=D \times E$ with $E=E^{\gamma}$, then $\gamma$ acts trivially on $E \simeq_{\gamma} A / D$ and therefore on the socle $\langle a, b\rangle$ of $A$, a contradiction.

On the other hand, if ( $i$ ), (ii) and (iii) in Definition 1 hold with only $A / D$ in the place of $E$, then $\gamma$ is neverthless an almost power automorphism and there is a finite $C \leq A$ and a subgroup $B$ such that $\gamma$ is either power or pseudo-power on both $A / C$ (see Theorem 2.11 in [2]) and $B$. The order of $C$ and the index $A / B$ are restricted, as in the following statement. 
Proposition 4. Let $p$ be a prime, $r, \varepsilon \in \mathbb{N}, e=p^{\varepsilon}, s \in Z$ and $\alpha a p$ adic. If $A$ is an abelian $(r, e)$-bounded $p$-group and $\gamma \in \operatorname{Aut}(A)$ is such that $\gamma_{\mid D}=\alpha, \gamma_{A / D}=s$ with $D:=D(A)$, then

i) $\gamma$ acts as a power or pseudo-power on $A / C$ where $C=D\left[p^{n}\right]$, with $p^{n}=\exp \left(\operatorname{ker}_{A / D}(\alpha-s)\right)$ and in particular

$$
|C|=p^{n r} \leq e^{r} ;
$$

ii) $\gamma$ acts as a power or pseudo-power on a subgroup $B$, with

$$
|A / B| \leq e^{r} .
$$

The above bounds are best-possible, as there exist abelian $(r, e)$-bounded p-groups $A$ and $\gamma \in$ Aut $A$ acting as power on $D=D(A)$ and $A / D$ such that

iii) if $\gamma$ acts as a power or pseudo-power on a factor $A / C$, then $C$ contains $D\left[p^{n}\right]$, where $\gamma_{\mid D}=\alpha$ and $p^{n}=\exp \left(\operatorname{ker}_{A / D}(\alpha-\gamma)\right)$, hence

$$
|C| \geq p^{n r}
$$

iv) if $\gamma$ acts as a power or a pseudo-power on a subgroup $B \leq A$, then

$$
|A / B| \geq e^{r} .
$$

Proof. $i$ ) Let $K$ and $E$ be the kernels of the endomorphisms $\alpha-s$ and $\gamma-s$ of $A$, resp. Clearly $n \leq \varepsilon$ and, even in case $n=\varepsilon$, we may assume $K \cap D=E \cap D=D\left[p^{n}\right]$, as we can substitute $s$ by $s+p^{\varepsilon}$. Obviously $D \geq A^{\gamma-s}$, and since $\gamma=s$ on no nontrivial images of $D$, we have that $A^{\gamma-s}=D$. As $A / E \simeq A^{\gamma-s}$, we get $A=E D$. Thus $\gamma$ is pseudo-power on $A / D\left[p^{n}\right]$.

ii) Let $A=D \times E$ and let $A_{1}=A[e]=D[e] \times E$. Then $\gamma=s$ on $A_{1} / D[e] \simeq_{\gamma} A / D$, so that $\gamma=s$ on $E_{1} \leq A_{1}$ with index at most $|D[e]|=e^{r}$. The statement holds with $B=D \times\left(E \cap E_{1}\right)$, as $|A / B|=\left|E: E \cap E_{1}\right| \leq e^{r}$.

iii) Let $A=A_{1} \times \cdots \times A_{r} \times E$, where for $0 \leq i \leq r, A_{i}=D_{i} \times\left\langle e_{i}\right\rangle$, with $D_{i}$ a Prüfer $p$-group, $e_{i}$ of order $e$ and $E$ an infinite elementary abelian $p$-group. Let $d_{i}$ be an element of order $e$ in $D_{i}$ for each $i$ and $\gamma \in$ Aut $A$ such that $\gamma=\alpha$ on $D=D_{1} \times \cdots D_{r}, \gamma=s$ on $E$ and $e_{i}^{\gamma}=e_{i}^{s} d_{i}$.

Let now $C$ be a $\gamma$-invariant subgroup of $A$ such that $A / C=$ $D C / C \times V / C$ and $\gamma=s$ on $V / C$. For $1 \leq i \leq r$, there exist $v_{i} \in V$ and $z_{i} \in D$ such that $e_{i}=v_{i} z_{i}$. Observe that $v_{i}^{e} \in C$, as $A / D$ has exponent $e$, and so $z_{i}^{e} \in C$. Now, as $\gamma=s$ on $V / C$, we get $d_{i} z_{i}^{s-\alpha} \in C$ and so $d_{i}^{p^{p-n}} \in C$. Therefore $C \geq D\left[p^{n}\right]$. 
iv) Let $A_{1}:=P \times V$, where $P$ is a Prüfer $p$-group and $V=\langle v\rangle$ has order $e=p^{\varepsilon}$. Define $\gamma$ by $\gamma=1+p$ on $P$ and $v^{\gamma}=v d$, where $d$ is an element of $P$ of order $p^{\varepsilon}$. Let $A^{\prime}$ be the direct product of $r$ copies $A_{i}$ of $A_{1}$ and define $\gamma$ on $A^{\prime}$ in the natural way componentwise. We show that:

$$
\text { (*) } \forall z \in A^{\prime} \backslash 1 \quad z^{\gamma}=z \Rightarrow\langle z\rangle \cap D \neq 1 .
$$

In fact, if $r=1$ we have that if $z=v^{n} d^{m}$ is such that the order of $d^{m}$ is not bigger than that of $v^{n}$. Then $n=p^{\bar{n}} n_{1}$ and $m=p^{\bar{m}} m_{1}$, with $p \notin \pi\left(n_{1} m_{1}\right)$ and $\bar{m} \geq \bar{n}$. As $z^{\gamma}=z$, we get $d^{n+p m}=1$, which implies $p^{\varepsilon}$ divides $n$ hence $v^{n}=d^{m}=1$ and $z=1$, a contradiction.

In the general case, $z=a_{1} \ldots a_{r}$, with $a_{i}=a_{i}^{\gamma} \in A_{i} \forall i$, which implies that $a_{i}=1$ or a nontrivial power $a_{i}^{s_{i}}$ of $a_{i}$ is in $D$, by the above. Choosing the biggest $s_{i}$ we get that $z^{s_{i}}$ is in $D \backslash 1$. Thus ( $*$ ) is proved.

Let now $A=A^{\prime} \times E$ where $E$ is an infinite elementary abelian $p$-group and extend $\gamma$ by $\gamma=1$ on $E$. Then $\gamma$ is power on both $D=D(A)$ and $A / D$.

Let $B$ be a subgroup of finite index of $A$ such that $\gamma$ acts as a pseudopower on $B$. Then $B=D \times Z$ and $\gamma=1$ on $Z$. By (*) we have that $Z \cap A^{\prime}=1$. Let $z=a^{\prime} h \in Z$ with $a^{\prime} \in A^{\prime}$ and $h \in E$. Then $z^{p} \in A^{\prime} \cap Z=1$. Then $a^{\prime} \in D$ has order $p$. Hence $z \in D E$ and so $B \leq D E$. It follows $|A / B| \geq e^{r}$.

Now we point out a property of pseudo-power automorphisms corresponding to that in Proposition 2.

Proposition 5. Let $p$ be a prime, $r, \varepsilon \in \mathbb{N}, e=p^{\varepsilon}, A$ be an abelian $(r, e)$-bounded p-group and $\gamma \in \operatorname{Aut}(A)$, then

i) If $\gamma$ is pseudo-power on a finite index subgroup $B$, then $\gamma$ is pseudo-power on a factor $A / C$ with both $|C| \leq|A / B| e^{r}$ and $|C| \leq|A / B|^{2}$. ii) If $\gamma$ is pseudo-power on a factor $A / C$ with $C$ finite, then $\gamma$ is pseudo-power on a subgroup $B$ with $|A / B| \leq|C| e^{r}$.

Consideration of the group in the proof of Proposition 4.iv shows that in Proposition 5.ii $|A / B|$ cannot be bounded in terms of $|C|$ only (take $C:=D[p])$.

Proof. $i$ ) Let $B=D \times E$ where $\gamma$ is power on both $D$ and $E$. Also, $A=D \times E_{1}$ for some $E_{1} \geq E$. Let $E_{2}:=E_{1}^{\langle\gamma\rangle}$ and $C_{2}:=D \cap E_{2}=C_{2}^{\gamma}$. By applying Proposition 2 to the the group $E_{2} / C_{2}$, we know that $\gamma$ is power on a factor $E_{2} / C$ such that $\left|C / C_{2}\right| \leq|A / B|$ and therefore $\gamma$ is pseudo-power on $A / C$. 
On one hand, $C_{2} \simeq E_{2} / E$ has rank at most $r$ and $\exp \left(E_{2} / E\right)=$ $\exp \left(E_{1} / E\right)$ divides $e$ hence $\left|C_{2}\right| \leq e^{r}$. On the other hand, by applying Proposition 2 to the group $A / E$ we have that $\gamma$ is power on a factor $A / C_{3}$ with $\left|C_{3} / E\right| \leq|A / B|$. Hence $E_{2} \leq E_{1} C_{3}$, so that $\left|C_{2}\right|=\left|E_{2} / E_{1}\right| \leq\left|C_{3} / E\right| \leq$ $|A / B|$.

ii) Let $A / C=D^{\prime} / C \times E / C$, where $D^{\prime} / C$ is divisible, $E$ has finite exponent and $\gamma$ is power on both $D^{\prime} / C$ and $E / C$. Observe that $D^{\prime}=D C$. Hence $A=D E$ and $\left|D^{\prime}\right| D|\leq| C \mid$. Moreover $\gamma$ acts as a power on $A / D^{\prime} \simeq_{\gamma} E / C$, then by Proposition $2, \gamma$ acts as a power automorphisms on a subgroup $A_{1} / D$ of index at most $|C|$ in $A / D$. The statement holds by Proposition 4.ii.

Let's recall now a key result, basically due to D. Robinson [7].

LEMma 1. Let $a \in A$ an abelian group and $\gamma$ an inertial automorphism. Then

i) $\gamma$ acts as a power automorphism on any divisible torsion subgroup of $A$;

ii) if a is periodic, then $a^{\langle\gamma\rangle}$ is finite.

Lemma 2. Let $A$ be an abelian p-group, $\gamma \in \operatorname{Aut}(G)$ and $m \in \mathbb{N}$. If $\left|X /\left(X \cap X^{\gamma}\right)\right| \leq p^{m}$ for all finite $X \leq A$, then $\left|X^{\langle\gamma\rangle} / X\right| \leq p^{2 m}$ for all finite $X \leq A$.

In Theorem 1 we will see that a similar statement holds even if the word "finite" is omitted.

Proof. Fix $X$ with order $p^{\varepsilon}$. We first prove the statement when $X=\langle a\rangle$ is cyclic. If $\varepsilon=1$, that is $X^{\langle\gamma\rangle}=\langle a\rangle^{\langle\gamma\rangle}$ is an elementary abelian $p$ group, consider the epimorphism $g \in Z_{p}[x] \mapsto a^{g(\gamma)} \in\langle a\rangle^{\langle\gamma\rangle}$ of $Z_{p}$-modules. By Lemma 1, $\langle a\rangle^{\langle\gamma\rangle}$ has finite order $p^{n}$ and so there is $f \in Z_{p}[x]$ with degree $n$ such that

$$
\frac{Z_{p}[x]}{(f)} \simeq_{\gamma}\langle a\rangle^{\langle\gamma\rangle}
$$

If $n \leq 2 m+1$ the statement is true. Assume false by contradiction. Then $X=\left\langle a, a^{\gamma^{2}}, \ldots, a^{\gamma^{2 m}}\right\rangle$ has order $p^{m+1}$. It follows that there is $b \in X \cap$ $X^{\gamma} \backslash\{1\}$. Thus there are polynomials $g, h \in Z_{p}[x]$ with degree $\leq m$ such that $b=a^{g\left(\gamma^{2}\right)}=a^{\gamma h\left(\gamma^{2}\right)}$. Therefore $f(x)$ divides $g\left(x^{2}\right)-x h\left(x^{2}\right)$ which is not the constant 0 and has degree $\leq 2 m+1$, a contradiction. 
If $\varepsilon>1, B:=\left\langle a^{p^{\varepsilon-1}}\right\rangle^{\langle\gamma\rangle}$ has order at most $p^{(2 m+1)}$, by case $\varepsilon=1$, and $\langle a\rangle^{\langle\gamma\rangle} / B$ has order at most $p^{(\varepsilon-1)(2 m+1)}$, by induction. The claim holds.

In the general case let $X=\left\langle a_{1}\right\rangle \times \cdots \times\left\langle a_{r}\right\rangle$ with $a_{i}$ of order $p^{\varepsilon_{i}}$ and $\varepsilon_{1}+\cdots+\varepsilon_{r}=\varepsilon$. By the above, for each $i$ we have $\left|\left\langle a_{i}\right\rangle^{\langle\gamma\rangle}\right| \leq p^{(2 m+1) \varepsilon_{i}}$ and $\left|X^{\langle\gamma\rangle} / X\right| \leq p^{2 m \varepsilon}$.

Let's show that for periodic abelian groups all properties $(a p),(b p),(i n)$, (bin) are equivalent. Recall that $(a p)$ and $(b p)$-automorphisms of abelian groups have been independently characterized in [3] and [2], respectively.

THEOREM 1. Let $A$ be a periodic abelian group and $\gamma \in \operatorname{Aut}(A)$. Then the following are equivalent to the fact that $\gamma$ is inertial:

i) $X /\left(X \cap X^{\gamma}\right)$ is finite for all $X \leq A$;

a) there exists $a \in \mathbb{N}$ such that $\left|X /\left(X \cap X^{\gamma}\right)\right| \leq$ a for all $X \leq A$;

$b)$ there is subgroup $B \leq A$ with finite index $b$, such that $\gamma$ is power on all but finitely many primary components of $B$ and pseudo-power on the other ones;

c) there is a subgroup $C \leq A$ with finite order $c$, such that $\gamma$ is power on all but finitely many primary components of $A / C$ and pseudopower on the other ones;

d) there exists $d \in \mathbb{N}$ such that $\left|X^{\langle\gamma\rangle} / X\right| \leq d$ for all $X \leq A$;

$\left.d_{*}\right)$ there exists $d_{*} \in \mathbb{N}$ such that $\left|X / X_{\langle\gamma\rangle}\right| \leq d_{*}$ for all $X \leq A$.

Moreover, there are functions (depending on $A$ ) bounding each of $a, b$, $c, d, d_{*}$ in terms of any other.

Proof. If $p$ is a prime greater that one among the above $a, b, c, d, d_{*}$, then the corresponding statement tells that $\gamma$ acts as a power automorphism on the $p$-component of $A$. Thus, according to Proposition 1, we assume that $A$ is a $p$-group. Let $D=D(A)$ and $r$ and $e$ the rank of $D$ and the exponent of $A / D$, respectively (where this cardinals are possible infinite).

First of all recall that:

$(c) \Leftrightarrow(b)$ by Propositions 2 and 5 . On the other side, if $A$ is periodic, (b) is equivalent to $(a p)$ (by Theorem 2.8 of [3] with $\Gamma=\langle\gamma\rangle$ ) and $(c)$ is equivalent to $(b p)$ (see Theorem 2.11 of [2]).

(i) $\Rightarrow(a p)$ Assume first $A$ has exponent $p$. We show that $\gamma$ is periodic (and so an almost power automorphism). By Lemma 1, the orbits induced by $\gamma$ are finite. Assume, by contradiction, they have unbounded orders. Let's show that for all finite $X \leq A$ such that $X \cap X^{\gamma}=1$ there exists $X_{1}>X$ with the same property. Note that $X^{\langle\gamma\rangle}$ is finite by Lemma 1 and 
choose $a \in A \backslash X^{\langle\gamma\rangle}$ whose orbit under the action of $\gamma$ has length larger than the order of $K:=\langle a\rangle X^{\langle\gamma\rangle}$. Thus $a^{\gamma} \notin K$ otherwise $\langle a\rangle^{\langle\gamma\rangle} \leq K$ contradicting the choice of $a$. Let now $X_{1}:=\langle a\rangle X$. We will show that $X_{1} \cap X_{1}^{\gamma}=1$. Let $y \in X_{1} \cap X_{1}^{\gamma}$. Hence $\exists n, s \in Z, \exists x, x_{0} \in X$ such that $y=a^{n} x=a^{s \gamma} x_{0}^{\gamma}$. Then $a^{s \gamma} \in\langle a\rangle X^{\langle\gamma\rangle}=K$ while $a^{\gamma} \notin K$. Therefore $a^{s}=1$ and it follows $a^{n} \in X^{\langle\gamma\rangle}$ while $a^{\gamma} \notin X^{\langle\gamma\rangle}$. Therefore $a^{n}=1$ as well. It follows $y=x=x_{0}^{\gamma} \in X \cap$ $X^{\gamma}=1$, as claimed. Now we define by induction a sequence of finite subgroups $X_{i}$ such that $X_{i} \cap X_{i}^{\gamma}=1$ and $X_{i}<X_{i+1}$. Thus $X_{\omega}:=\bigcup_{i} X_{i}$ is infinite and $X_{\omega} \cap X_{\omega}^{\gamma}=1$, a contradiction. Thus $\gamma$ acts as an (ap)-automorphism on the socle $A[p]$ of $A$.

Let $X$ be any subgroup of $A$. To show that $X / X_{\langle\gamma\rangle}$ is finite, we may suppose $X_{\langle\gamma\rangle}=1$. Thus, since $\gamma$ acts as a power automorphism on $D$ (see Lemma 1), we have $D \cap X=1$ and $X$ is reduced. On the other hand, since we have seen that $\gamma$ is almost power on $A[p]$ we get that $X[p]$ is finite. It follows that $X$ is finite.

$(a) \Rightarrow(d)$ Apply Lemma 2 to the group $A / X_{\langle\gamma\rangle}$ and get that

$$
\left|X^{\langle\gamma\rangle} / X\right| \leq a^{2} .
$$

$(d) \Rightarrow(c)$ Trivially $(d)$ implies $(a)$. Therefore $\gamma$ is $(a p)$ and, if $A$ is reduced, by the above there is a power automorphism $\alpha$ of $A$ such that $A^{\gamma-\alpha}$ has finite order. In order to find a bound for $c$ depending on $d$, let us show that:

if $A$ is reduced, then $\left|A^{\gamma-\alpha}\right| \leq d^{\frac{2^{2}+3 e}{2}}$ or $\leq d$, according to the fact that $\exp (A)=p^{\varepsilon}<\infty$ or $=\infty$, respectively .

Let first $\exp (A)<\infty$. By contradiction, fix the smallest $\varepsilon \in \mathbb{N}$ for which the statement is false, where clearly $\varepsilon>0$. We have that $\gamma$ is power on no factor $A / C$ with $|C| \leq c(d, \varepsilon):=d^{\frac{2^{2}+3 \varepsilon}{2}}$. Then fix the largest $n \in \mathbb{N}$ such that

$$
\exists A_{n} \leq A \quad: \quad\left|A_{n}\right|=p^{\varepsilon n} \quad \text { and } \quad\left|A_{n}^{\langle\gamma\rangle} / A_{n}\right| \geq p^{n}
$$

where the above holds for $n=0$, at least. Denote $C_{n}:=A_{n}^{\langle\gamma\rangle}$. We have

$$
p^{(\varepsilon+1) n} \leq\left|C_{n}\right| \leq\left|A_{n}\right| d \leq p^{\varepsilon n} d \quad \text { and } \quad p^{n} \leq d .
$$

The subgroup $A^{\#}=\left\{a \in A \mid a^{p^{\varepsilon-1}} \in C_{n}\right\}$ is such that $\exp \left(A^{\#} / C_{n}\right)<p^{\varepsilon}$. Thus, by the choice of $\varepsilon$, there exists $C$ such that $C_{n} \leq C \leq A^{\#}, \gamma$ acts as a power on $A^{\# / C}$ and

$$
|C|=\left|C_{n}\right| \cdot\left|C / C_{n}\right| \leq p^{\varepsilon n} d \cdot c(d, \varepsilon-1) \leq d^{\varepsilon+1} \cdot c(d, \varepsilon-1)=c(d, \varepsilon)
$$

Let us show now that $\gamma$ acts as a power on $A / C$, that is for all $a \in A$ we have 
$a^{\gamma} \in C\langle a\rangle$. If $a \in A^{\#}$, then the thing is true by the above. Otherwise $a$ has order $p^{\varepsilon}$ even modulo $C_{n}$, by definition of $A^{\#}$. In particular $\langle a\rangle \cap C_{n}=$ $\langle a\rangle \cap A_{n}=1$ and $A_{n+1}:=A_{n}\langle a\rangle=A_{n} \times\langle a\rangle$ has order $p^{n+1}$. If $a^{\gamma} \notin C\langle a\rangle$, then $a^{\gamma} \notin C_{n}\langle a\rangle$ so that

$$
p^{n} \leq\left|C_{n} / A_{n}\right|=\left|C_{n}\langle a\rangle / A_{n}\langle a\rangle\right|<\left|A_{n+1}^{\langle\gamma\rangle} / A_{n+1}\right|
$$

contradicting the choice of $n$.

Let now $\exp (A)=\infty$ and $A$ be residually finite. We see that there is an $X$ such that

$$
X \cap A^{\gamma-\alpha}=1 \quad \text { and } \quad A^{\gamma-\alpha} \leq X^{\langle\gamma\rangle}
$$

whence $\left|A^{\gamma-\alpha}\right| \leq\left|X^{\langle\gamma\rangle} / X\right| \leq d$. To do this, proceed by induction and assume that for a maximal subgroup $C_{1}$ of $C:=A^{\gamma-\alpha}$ there exist $X_{1}$ such that $X_{1} \cap C=1$ and $C_{1} \leq X_{1}^{\langle\gamma\rangle}$. Let $\alpha^{\gamma-\alpha} \in C \backslash C_{1}$. As $A$ is residually finite and $\gamma$ is (ap) on $A$, by the above $\gamma=\alpha$ is power on a subgroup $B=B^{\langle\gamma\rangle}$ with finite index in $A$ and such that $B \cap C X_{1}\langle a\rangle=1$. As $A$ as infinite exponent, the same holds for $B$ and we can pick and element $b \in B$ with the same order as $a$. We have then $a^{\gamma-\alpha}=(a b)^{\gamma-\alpha}$, thus $C=C_{1}\left\langle a^{\gamma-\alpha}\right\rangle \leq X^{\langle\gamma\rangle}$ where $X:=X_{1}\langle a b\rangle$. Further if $c \in X \cap C$, then $c=x_{1} a^{m} b^{m}$ for some $x_{1} \in X_{1}, m \in \mathbb{N}$, so that $b^{m} \in B \cap C X_{1}\langle a\rangle=1$. By the choice of $b$, we have $a^{m}=1$ as well. It follows $c=x_{1} \in X_{1} \cap C=1$, as desired. The case $A$ is residually finite is now complete.

In the case $A$ is reduced, but perhaps not residually finite, let $B$ be a basic subgroup of $A$ and $B_{1}=B^{\langle\gamma\rangle}$. Then $B_{1} / B$ is finite, so that $B_{1}$ is residually finite and $A / B_{1}$ is divisible. By the above there exists a subgroup $C \leq B_{1}$ with order $\bar{c} \leq d^{\frac{\varepsilon^{2}+3 \varepsilon}{2}}$ or $d$ (according to $\exp (A)=p^{\varepsilon}<\infty$ or $=\infty$ ), such that $\gamma=\alpha$ on $B_{1} / C$. Then the kernel of $\gamma-\alpha$ on $A / C$ contains $B_{1} / C$, so that the image is divisible. On the other hand $A / C$ is reduced, so that $\gamma=\alpha$ on $A / C$.

In the general case, let $A=D \times R$, where $R$ is reduced. Then $C_{1}=R^{\langle\gamma\rangle} \cap D$ has order at most $d$. On one hand, by the above, the reduced group $R^{\langle\gamma\rangle} / C_{1}$ has a subgroup $C / C_{1}$ of order at most the above $\bar{c}$ such that $\gamma$ is power on $R^{\langle\gamma\rangle} / C$. On the other hand $\gamma$ is power on the divisible group $D C / C$ as well, so that $\gamma$ is either power or pseudo-power on $A / C$. Clearly, according to $\exp (A)=p^{\varepsilon}<\infty$ or $=\infty, C$ has order

$$
c \leq d^{\frac{\varepsilon^{2}+3 \varepsilon+2}{2}} \text { or } d^{2} \text {. }
$$

$(b) \Rightarrow\left(d_{*}\right)$. Let $X$ be any subgroup of $A$. Then by Proposition 3, $\left|(X \cap B) /(X \cap B)_{\langle\gamma\rangle}\right| \leq e^{r}$. Thus $\left(d_{*}\right)$ holds with

$$
\left|X / X^{\langle\gamma\rangle}\right| \leq b e^{r} .
$$

$\left(d_{*}\right) \Rightarrow(a)$ This is trivial. 
In the notation of Theorem $1, d_{*}$ is bounded by $b e^{r}$ and clearly $d$ is bounded by $c e^{r}$. Let us show that these bounds are best possible.

Proposition 6. For each prime $p \neq 2, r, \varepsilon \in \mathbb{N}, e=p^{\varepsilon}$, there exists an abelian $(r, e)$-bounded p-group such that:

i) there is an inertial automorphism $\gamma$ acting as a pseudo-power on a finite index subgroup $B$ and a subgroup $X$ of $A$ such that

$$
\left|X / X_{\langle\gamma\rangle}\right|=|A / B| e^{r} .
$$

ii) there is an inertial automorphism $\gamma$ acting as a pseudo-power on a factor $A / C$ where $C$ is finite and a subgroup $X$ of $A$ such that

$$
\left|X^{\langle\gamma\rangle} / X\right|=|C| e^{r} .
$$

Proof. Let $A=D \times E \times C \times C_{0}$ be a $p$-group where $D$ is divisible with rank $r, E$ is the product of infinitely many copies of a cyclic group with order $e=p^{\varepsilon}, C_{0}=\left\langle c_{0}\right\rangle$ and $C$ elementary abelian with orders $p$ and $p^{\varepsilon}$, resp. Define, for the moment, $\gamma_{D E C}$ by $\gamma_{\mid D}=\alpha, \gamma_{\mid E}=1, \gamma_{\mid C}=\gamma_{1}$ where $\alpha$ a $p$-adic $\not \equiv 1(\bmod p), \gamma_{1}$ is an irreducibile automorphism of $C$ and $a$ such that $C=\langle a\rangle^{\left\langle\gamma_{1}\right\rangle}$. By Proposition 3 there is $X_{0} \leq D E$ such that $\left|X_{0} / X_{0\langle\gamma\rangle}\right|=e^{r}$ and $\left|X_{0}^{\langle\gamma\rangle} / X_{0}\right|=e^{r}$.

i) Define $\gamma$ on the whole $A$ by $\gamma_{\mid C_{0}}=1$. Clearly $\gamma$ is pseudo-power on $B:=D E C_{0}$. Let $X:=X_{0} M$ where $M$ is a maximal subgroup of $C_{0} C$ containing neither $C_{0}$ nor $C$. We have $X_{\langle\gamma\rangle}=X_{0\langle\gamma\rangle} \times M_{\langle\gamma\rangle}$, so that $\left|X / X_{\langle\gamma\rangle}\right|=$ $|A / B| e^{r}$.

ii) Define $\gamma$ on the whole $A$ by $c_{0}^{\gamma}=c_{0} a$. Clearly $\gamma$ is pseudo-power on $A / C$. If $X:=X_{0} C_{0}$ we have $\left|X^{\langle\gamma\rangle} / X\right|=|C| e^{r}$.

\section{Torsion-free case}

Recall $(a p)$ and $(b p)$-automorphism of abelian torsion-free groups are just the identity or the inversion map (see [3] and [2] or just later). The picture is different for (in)-automorphisms. In this section we use additive notation for the abelian group operation in $A$.

Notice that if $0 \neq n, m \in Z$, for each $a \in A$, a torsion-free abelian group, there is at most one $b \in A$ such that

$$
(*) \quad n b=m a .
$$


If such a unique $b$ exists, we write $b=\frac{m}{n} a$. Thus when $m, n$ are coprime, (*) defines an automorphism $a \mapsto b$ if and only if $A=m A=n A$. We say that this is the rational power automorphism $\frac{m}{n}$.

The following result describes the structure of the group $\mathcal{I} \operatorname{Aut}(A)$ of all inertial automorphisms of $A$ in terms of $A$, in the case $A$ is torsion-free. Denote $\bar{\pi}(A)$ the set of primes $p$ such that $p A=A$.

THEOREM 2. Let $(A,+)$ be an abelian torsion-free group.

If $\gamma \in \mathcal{I} \operatorname{Aut}(G)$, then $\gamma: x \mapsto \frac{m}{n} x$ with $m, n$ coprime integers and $\pi(m n) \subseteq \bar{\pi}(A)$.

If $\mathcal{I} \operatorname{Aut}(A) \neq \operatorname{PAut}(A)=\{ \pm 1\}$, then $A$ has finite rank $r$ and

$$
\left\langle a_{1}, \ldots, a_{s}\right\rangle^{\langle\gamma\rangle} \simeq \mathrm{Q}_{\pi} \oplus \ldots \oplus \mathbb{Q}_{\pi} \quad(s \text { times })
$$

where $\pi=\pi(m n)$, whenever the $a_{i}$ 's are Z-independent elements of $A$. Further, if $\pi(m n) \subseteq \bar{\pi}(A)$ then $\frac{m}{n} \in \mathcal{I} \operatorname{Aut}(A)$ so that

$$
\mathcal{I} \operatorname{Aut}(A) \simeq\{+1,-1\} \times \operatorname{Dr}_{p \in \bar{\pi}(A)}\langle p\rangle
$$

Proof. For any $a \in A$, since $\left|\langle a\rangle:\langle a\rangle \cap\left\langle a^{\gamma}\right\rangle\right|=m \in \mathbb{N}$, there exists an $m \in Z$ such that $m a=n a^{\gamma}$. As $A$ is torsion-free, $m, n$ can be choosen coprime. Let us show that $\frac{m}{n}$ does not depend on $a$. Let $a_{1} \in A$. If $\left\langle a_{1}\right\rangle \cap\left\langle a^{\gamma}\right\rangle \neq\{0\}$, then $a_{1}=\frac{h}{k} a$ for some $h, k \in \mathbb{Z}$. Therefore

$$
a_{1}^{\gamma}=\frac{h}{k} a^{\gamma}=\frac{h}{k} \frac{m}{n} a=\frac{m}{n} a_{1}
$$

Similarly, if $\left\langle a_{1}\right\rangle \cap\left\langle a^{\gamma}\right\rangle=\{0\}$, there will exist $m_{1}, m_{2}, n_{1}, n_{2} \in Z_{\text {such }}$ that

$$
\frac{m}{n} a+\frac{m_{1}}{n_{1}} a_{1}=a^{\gamma}+a_{1}^{\gamma}=\left(a+a_{1}\right)^{\gamma}=\frac{m_{2}}{n_{2}}\left(a+a_{1}\right)=\frac{m_{2}}{n_{2}} a+\frac{m_{2}}{n_{2}} a_{1} .
$$

It follows $\frac{m}{n}=\frac{m_{2}}{n_{2}}=\frac{m_{1}}{n_{1}}$ and $\gamma \mapsto \frac{m}{n}$ is a monomorphism $\mathcal{I} \operatorname{Aut}(A) \rightarrow Q^{*}$, the multiplicative group of the rationals.

Observe now that for each $X \leq A$, by applying the monomorphism $x \mapsto n x$ we have $X / X \cap X^{\gamma} \simeq n X / n X \cap m X$. The latter has exponent at 
most $m$ and is finite if $X$ has finite rank. Conversely, if the rank of the whole $A$ is infinite, we get a contradiction by taking $X$ to be free of maximal rank.

Finally, if the rank of $A$ is $r$, then $A$ is isomorphic to a subgroup $B$ of $\mathrm{Q} \oplus \ldots \oplus \mathrm{Q}(r$ times $)$ containing $\mathrm{Z} \oplus \ldots \oplus \mathrm{Z}(r$ times $)$. Since $B$ is $\bar{\pi}$-divisible, it is clear that $B$ contains $\mathrm{Q}_{\bar{\pi}} \oplus \ldots \oplus \mathrm{Q}_{\bar{\pi}}(r$ times), where $\bar{\pi}=\bar{\pi}(A)$.

Recall that in Theorem 1 we have seen that $X /\left(X \cap X^{\gamma}\right)<\infty$ for each $X \leq A$ implies $\gamma$ is inertial indeed, provided $A$ is periodic. However, this is no longer true if $A$ is non-periodic, a counterexample being the map $\gamma: x \mapsto \frac{1}{2} x$ in the product of infinitely many copies of $\mathrm{Q}$ (see Theorem 2 ).

\section{Mixed case}

We start by observing that, by Theorem 2 , if $A$ is non-periodic with torsion subgroup $T$ and $\gamma$ is inertial then there exist coprime $m, n \in Z$ such that

$$
\gamma_{\mid S}=\gamma_{\mid A / T}=\frac{m}{n}
$$

for each torsion-free $\gamma$-invariant section $S$ of $A$. Define $\pi(\gamma):=\pi(m n)$.

Now we prove a generalization of Lemma 1 . Recall that a p-group has finite exponent iff it has no nontrivial divisible quotients. (see Cor. 35.4 in [4]).

LEMma 3. Let $\gamma$ an inertial automorphism of an abelian group A. If $a \in A$, then the torsion subgroup $T$ of $V=\langle a\rangle^{\langle\psi\rangle}$ is finite. Thus there is $e \in \mathbb{N}$ such that $V^{e} \simeq \mathrm{Q}_{\pi(\gamma)}$ has finite index in $V$, provided a is aperiodic.

Proof. We can assume $A=V$. Let $\gamma=\frac{m}{n}$ on $A / T$. Define inductively $A_{0}:=\langle a\rangle$ and $A_{i+1}:=A_{i} A_{i}^{\gamma} A_{i}^{\gamma^{-1}}$. Then $A=\bigcup_{i<\omega} A_{i}$ where, for each $i, A_{i+1} / A_{i}$ is generated by its elements $a^{\gamma^{i}} A_{i}$ and $a^{\gamma^{-i}} A_{i}$ with order dividing $m$ and $n$, respectively. Thus $T \simeq T A_{0} / A_{0}$ and $\pi(T) \subseteq \pi\left(A / A_{0}\right)$ consists of divisors of $m n$. In particular $\pi(T) \subseteq \pi(m n)$ is finite.

Suppose now $A$ splits on $T$, that is $A=T \times B$ for some $B$. Let then $B_{1}=B B^{\gamma} B^{\gamma^{-1}}$ and note that $B_{1} / B$ is finite. Then $B_{1}=T_{1} \times B$, where $T_{1}$ is finite subgroup of $T$. Thus by Lemma $1, K=T_{1}{ }^{\langle\gamma\rangle}$ is finite as well. Moreover $(B K)^{\gamma}=B^{\gamma} K \leq B_{1} K=B K$ and similarly $(B K)^{\gamma^{-1}} \leq B K$. Thus $B K$ is 
$\gamma$-invariant. Therefore $T / K \simeq A / B K$ is finite, by Lemma 1 again. Thus $T$ is finite, in this case, which applies if $T$ has finite exponent.

If, by contradiction, $T$ has infinite exponent there is a factor $T / R$ which is a Prüfer $p$-group. Since $R^{\langle\gamma\rangle} / R$ is finite, by Theorem 1 , then $T / R^{\langle\gamma\rangle}$ is a Prüfer group and $A / R^{\langle\gamma\rangle}$ splits on its torsion subgroup $T / R^{\langle\gamma\rangle}$ which turns out to be finite, by the above, a contradiction.

Finally, if $e$ is the order of $T$, we have $A^{e}=\left\langle a^{e}\right\rangle^{\langle\gamma\rangle}$ is torsion-free and has finite index in $A$ by Lemma 1.

We come now to a characterization of inertial automorphisms of an abelian group. Before we state some elementary facts as a Lemma.

LEMma 4. Let $B$ a subgroup of an abelian group $A$ and $\pi$ a set of primes.

i) If $A / B$ is a $\pi$-group, then $A$ is $\pi$-divisible iff $B$ is $\pi$-divisible.

ii) If $A$ if torsion-free, $A / B$ periodic and $B$ is $\pi$-divisible then $A / B$ is a $\pi^{\prime}$-group and $A$ is $\pi$-divisible.

THEOREM 3. Let $\gamma$ be an automorphism of an abelian non-periodic group $A$. Then $\gamma$ is inertial if and only if one of the following holds:

(a) there a $\gamma$-invariant finite index subgroup $B$ of $A$ on which $\gamma$ acts as a power.

(b) there are finitely many elements $a_{i}$ such that:

1. the subgroup $V=\left\langle a_{1}, \ldots, a_{r}\right\rangle^{\langle\gamma\rangle}$ is torsion-free and $\gamma$ induces on it a rational power automorphism $\frac{m}{n}$ where $m, n$ are coprime integers;

2. the factor group $A / V$ is torsion and $\gamma$ induces an almost power automorphism on it;

3. the $\pi(m n)$-component of the torsion subgroup $T$ of $A$ has finite exponent.

Further, $A^{n \gamma-m}$ is periodic. It has finite exponent iff

(*) $\quad$ there exists $k \in \mathbb{N}$ such that $\left|X /\left(X \cap X^{\gamma}\right)\right| \leq k, \forall X \leq A$.

In case (b), $V \simeq \mathrm{Q}_{\pi} \oplus \ldots \oplus \mathrm{Q}_{\pi}$ ( $r$ times), where $\pi=\pi(m n)$, and $\gamma$ is almost power iff $V$ is free abelian.

About case ( $(a)$ see also Corollary 2 above. Condition (2) is characterized in Theorem 1 while (3) is always true if $\gamma$ is almost power, as $\pi=\emptyset$ in this case. 
Proof. Let $T$ be the torsion subgroup of $A$. Suppose that $\gamma$ is inertial. By Theorem 1, $\gamma$ acts as an almost power automorphism on each periodic $\gamma$-section of $A$. In particular, $\gamma$ is almost power on $T$ and rational power $\frac{m}{n}$ on $A / T$ (with $m, n$ coprime integers).

If $\frac{m}{n}= \pm 1$, then $\gamma$ is an almost power automorphism of $A$. To see this, let $S$ be a maximal independent subset of elements of infinite order of $A$. Then $Y=\langle S\rangle=\operatorname{Dr}_{s \in S}\langle s\rangle$ is free abelian and $A / Y$ is periodic. Let $s \in S$. By Lemma 3, the torsion subgroup $R$ of $\langle s\rangle^{\langle\gamma\rangle}$ is finite and so $\langle s\rangle^{\langle\gamma\rangle} /\langle s\rangle$ is finite too, since $\gamma$ is power on $\langle s\rangle^{\langle\gamma\rangle} / R$. Hence $\langle s\rangle$ has a subgroup of finite index $Y_{s}$ which is $\gamma$-invariant and so the subgroup $Z=\operatorname{Dr}_{s \in S} Y_{s}$ is free abelian and $\gamma$-invariant, $A / Z$ is periodic and $\gamma$ acts as a power automorphism on $Z$. If $Z$ has finite rank we are in case $(b)$ of Theorem 2.10 of [3] (with $\Gamma=\langle\gamma\rangle$ ) and $\gamma$ is almost power on $A$ and $(b)$ in the statement holds. On the other hand, if $Z$ has infinite rank, then $(a)$ holds. This can be shown by the same argument as in the proof of Theorem 2.10 of [3], keeping in mind that $\gamma$ acts as an almost power automorphism already on each periodic factor of $A$. The claim is proved.

If $\frac{m}{n} \neq \pm 1$, by Theorem 2 a maximal independent subset $\left\{s_{1}, \ldots, s_{r}\right\}$

of elements of infinite order of $A$ has finite cardinality. Thus by Lemma 3 there is $e \in \mathbb{N}$ such that $V:=\left\langle s_{1}^{e}, \ldots, s_{r}^{e}\right\rangle^{\langle\gamma\rangle}$ is torsion-free of rank $r$. Thus (1) and (2) hold.

Let us show that the $\pi(m n)$-component of $T$ has finite exponent. Assume, by contradiction, that $T$ has an image $T / X$ isomorphic to a Prüfer $p$-group, with $p \in \pi(m n)$. By Theorem $1, X^{\langle\gamma\rangle} / X$ is finite. Thus, without loss of generality, we may assume that $X^{\langle\gamma\rangle}=1$ and $A=V \times T$. Also we can take $1 \neq v \in V$ and assume $V=\langle v\rangle^{\langle\gamma\rangle}$ has rank 1 and is $\pi$-divisible. Then $A=V \times T$ where $T=\left\langle c_{n} \mid n \in \mathbb{N}_{0}, c_{0}=1, c_{n+1}^{p}=c_{n}\right\rangle$ is a Prüfer $p$-group. Consider then the subgroup $L_{1}$ of $A$ generated by all elements $v^{p^{-i}} c_{i}$, with $i \in \mathbb{N}$ (recall that $V$ is torsion-free and $p$-divisible, thus $v^{p^{-i}}$ is uniquely identified). Clearly $L_{1} /\langle v\rangle$ is the "diagonal subgroup" of the $p$-component of $A /\langle v\rangle$, which is the direct product $V_{p} /\langle v\rangle \times T\langle v\rangle /\langle v\rangle$ of two Prüfer $p$ groups. Also let $L_{2} /\langle v\rangle$ be the $p^{\prime}$-component of the whole $A /\langle v\rangle$ and finally $L=L_{1} L_{2}$. This is easily verified to be torsion-free, that is $L \cap T=1$. On one hand, $L \simeq L T / T=V T / T \simeq V$ and $L$ is $\pi$-divisible. On the other hand, $A / L \simeq T$ is a $p$-group, so that $L /\left(L \cap L^{\gamma}\right)$ is a $p$-group, which is finite as $\gamma$ is inertial. It follows $L=L^{\gamma}$. Therefore $\langle v\rangle=L \cap V=(L \cap V)^{\gamma}=\langle v\rangle^{\gamma}$, contradicting the fact that $\frac{m}{n} \neq \pm 1$. 
Finally suppose that $(*)$ holds and assume, by contradiction, that there exists $a \in A$ such that $b:=a^{n \gamma-m}$ has order greater that $k$. We can assume $a$ has infinite order (otherwise substitute with $a v$ where $1 \neq v \in V$ ). Let $X:=\langle a\rangle$. Thus $b \in X X^{\gamma}$ and therefore $\left|X X^{\gamma} / X^{\gamma}\right|>k$, a contradiction.

Conversely, note that if ( $a$ ) holds then $\gamma$ is (ap). Thus suppose that conditions (1), (2), (3) hold. Notice that $\gamma=\frac{m}{n}$ on $A / T$, that is $A^{n \gamma-m}$ is periodic.

We have to show that for each $X \leq A$ it holds $X \sim X^{\gamma}$ (where by $\sim$ we denote commensurability). We immediately notice that this is true if $X$ is periodic. In fact, in such a case $X V \sim X^{\gamma} V$ and $X /\left(X \cap X^{\gamma}\right) \simeq$ $X V /\left(X V \cap X^{\gamma} V\right)$ is finite, that is $X \sim X^{\gamma}$. Thus $\gamma$ acts as an inertial automorphism on $T$.

Let us show that when $A^{n \gamma-m}$ has finite exponent $e$ then

$$
\left|X /\left(X \cap X^{\gamma}\right)\right| \leq k:=d_{*}|m|^{r} e^{r}
$$

where $d_{*}$ is the bound given by Theorem $1 . d_{*}$. In fact for each $a \in A$ we have $a^{e n \gamma}=a^{e m}$. So that $X^{e n \gamma}=X^{e m} \leq X \cap X^{\gamma}$. Also $S_{\langle\gamma\rangle} \leq X \cap X^{\gamma}$, where $S$ is the torsion subgroup of $X$ and $\left|S / S_{\langle\gamma\rangle}\right| \leq d_{*}$. Now, on one hand $\left|X / X^{e m} S\right| \leq(e|m|)^{r}$, as $X / S$ is torsion-free with rank at most $r$. On the other hand $X^{e m} S / X^{e m} S_{\langle\gamma\rangle}$ is isomorphic to a factor of $S / S_{\langle\gamma\rangle}$ which has order at most $d_{*}$. Thus $\left|X / X^{e m} S_{\langle\gamma\rangle}\right| \leq d_{*}(e|m|)^{r}$, as claimed.

Let us treat the general case, where $T$ is possibly unbounded. We show that $X \sim X^{\gamma}$. We claim that we may reduce to the case $X$ is torsion-free, as if $S$ is the torsion subgroup of $X$, then $S / S_{\langle\gamma\rangle}$ is finite, by the above. So, we may factor out by $S_{\langle\gamma\rangle}$ (as conditions (1), (2), (3) are inherited by factors by torsion $\langle\gamma\rangle$-subgroups). Then we may assume that $S$ has finite order $s$ and $X^{s}$ is torsion-free. Since $X \sim X^{s}$, from now on assume $X$ is torsion-free.

We claim that we may factor out by any $\gamma$-invariant subgroup $E$ with finite exponent $e$. In fact, if $u=\left|X X^{\gamma} E / X E\right|$ is finite, then for any $x \in X$, $\left(x^{\gamma}\right)^{u} \in X E$. On the other hand $x^{n \gamma-m} \in T$ by the above. Thus $x^{u(n \gamma-m)} \in X E \cap T=E$, as $X$ is torsion-free. Therefore $x^{e u(n \gamma-m)}=1$ that is $x^{\text {eun } \gamma}=x^{\text {eum }}$. Hence $\left(X^{\text {eun }}\right)^{\gamma}=X^{\text {eum }}$. It follows $X^{\gamma} \sim\left(X^{\text {eun }}\right)^{\gamma}=X^{\text {eum }} \sim X$, as wished.

By taking $E=T_{\pi}$, we may assume that $T$ is a $\pi^{\prime}$-group. Moreover there are only finitely many primes $p$ for which the action of $\gamma$ on the $p$-component $T_{p}$ of $T$ is not power (Theorem 1). However, for each of these primes $p_{1}, \ldots, p_{l}$ there is a $\gamma$-invariant subgroup of finite exponent $E_{p_{i}}$ of $T_{p_{i}} \operatorname{such} \gamma$ acts as a power automorphism on $T_{p_{i}} / E_{p_{i}}$. So again we can take $R=E_{p_{l}} \cdots E_{p_{l}}$ and, by the above, assume that $\gamma$ acts as a power automorphism on $T$. 
Proceed now by induction on $r$, the torsion-free rank of $A$. Let $r=1$. Let $K / X$ be the $\pi$-component of $A / X$. Then $K$ is is torsion-free, as $T$ is a $\pi^{\prime}-$ group. By applying Lemma 4.(ii) to the group $A / T$ and to the subgroup $V T / T$, we get $A / T$ is $\pi$-divisible. Moreover, since $A / K T$ is a $\pi^{\prime}$-group by Lemma 4.(ii) we get $K \simeq K T / T$ is $\pi$-divisible. Let $b \in K \cap V$. Then for any prime $p \in \pi$ there exist $k \in K$ and $v \in V$ such that $k^{p}=b=v^{p}$. Hence $\left(k^{-1} v\right)^{p}=1$ so that $k=v \in K \cap V$, as $T$ is a $\pi^{\prime}$-group. It follows that $K \cap V$ is $\pi$-divisible and so has finite index in $V$, as $V$ has rank 1 .

Then $V K / K$ is finite. As $V K$ is inertized by $\gamma$, so is $K$. Therefore $K K^{\gamma} \cap T$ is finite and so $X X^{\gamma} \cap T$ is finite, too. Now $X X^{\gamma} /\left(X X^{\gamma} \cap X T\right) \simeq X X^{\gamma} T / X T$ is finite, and $\left(X X^{\gamma} \cap X T\right) / X \simeq X X^{\gamma} \cap T$ is finite as well. Hence $X \sim X^{\gamma}$.

Let now $r>1$ and let $r_{1}$ be the rank of $X$. If $r_{1}<r$, then $X^{\langle\gamma\rangle} T$ has rank $r_{1}$, and $X \sim X^{\gamma}$, by induction on $r$. If $X$ has rank $r, X$ can be written as $X_{1} X_{2}$, where both $X_{1}$ and $X_{2}$ have rank $<r$. Thus $\gamma$ inertizes both $X_{1}$ and $X_{2}$, then $X \sim X^{\gamma}$.

The last part of the statement follows from Theorem 2.10 of [3] and Theorem 2.

COROLLARY 1. Let $A$ be abelian group with torsion subgroup $T$ and $\gamma \in \operatorname{Aut}(A)$. Then the following are equivalent:

i) $\gamma$ is (ap), that is almost power,

ii) $\gamma$ is (bp),

iii) $\gamma$ is inertial on $A$ and power on $A / T$.

Consideration of $V$ in the statement of previuos theorem is necessary, even when $A$ splits on its torsion subgroup as we see in next Proposition.

Proposition 7. There is an abelian group $A$ with a non-inertial automorphism $\gamma$ which is inertial on both $T$ and $A / T$, where $T$ is the torsion subgroup of $A$.

Proof. Consider $A=D \times T$ with $D \simeq \mathrm{Q}_{6}, T \simeq Z_{2^{\infty}}, \gamma_{\mid T}=1$ and $\gamma_{\mid D}=3$. Then there is $V=V^{\gamma} \leq D$ with $V \simeq Q_{3}$. Hence $\gamma$ is not inertial on $A / V$, which is periodic and divisible, as it is not power on it.

Observe that there exist almost power automorphisms that are not boundedly inertial.

COROLLARY 2. There exists an inertial automorphism of an abelian group $A$ such that $\left|X /\left(X \cap X^{\gamma}\right)\right|$ is unbounded when $X \leq A$. 
PRoof. Let $n, m$ any coprime integers, with $\frac{m}{n} \neq 1$, let $\pi=\pi(n m)$ and consider the group $A=V \times T$, where $V$ is isomorphic to $\mathrm{Q}_{\pi}$ and $T$ is a periodic $\pi^{\prime}$-group with infinite exponent. Let $\gamma \in \operatorname{Aut}(A)$ such that $\gamma_{\mid V}=\frac{m}{n}$ and $\gamma_{\mid T}=1$. By Theorem 3, $\gamma$ is (in) and even (ap) when $\frac{m}{n}=-1$. Also $A^{n \gamma-m} \geq T^{n \gamma-m}=T^{n-m}$ has infinite exponent. Thus $\left|X /\left(X \cap X^{\gamma}\right)\right|$ is unbounded.

\section{Groups of inertial automorphisms}

Properties corresponding to ( $a p)$ and ( $b p)$ can be stated for a group $\Gamma$ of automorphisms of an abelian group, instead than for a singular $\gamma \in \operatorname{Aut}(A)$, as in [3] and [2], resp. So we define the following properties for $\Gamma$.

(AP) $\forall X \leq A \quad\left|X / X_{\Gamma}\right|<\infty$;

(BP) $\forall X \leq A \quad\left|X^{\Gamma} / X\right|<\infty$;

(CP) $\forall X \leq A \exists Y=Y^{\Gamma} \leq A$ such that $X$ and $Y$ are commensurable.

Obviously both $(A P)$ and $(B P)$ imply $(C P)$. From results in [3] (resp. [2]) it follows that $\gamma$ is $(a p)$ (resp. $(b p))$ iff $\langle\gamma\rangle$ is $(A P)$ (resp. $(b p)$ ). We will see in Proposition 9 that these three properties coincide if $\Gamma$ is finitely generated. On the other hand they are in fact different, as next Proposition 8 shows. Observe also that any of $(A P),(B P)$ or $(C P)$ for $\Gamma$ implies that $\Gamma \leq \mathcal{I} \operatorname{Aut}(A)$, where the latter is the subgroup of $\operatorname{Aut}(A)$ filled by all inertial automorphism.

Proposition 8. There exist infinite p-groups $A$ and $\Gamma \leq \operatorname{Aut} A$ which are:

(a) $A P$, not $B P$;

(b) $B P$, not $A P$;

(c) $\mathrm{CP}$, neither AP nor BP.

Proof. (a) Let $B=\operatorname{Dr}_{i \in \mathbb{N}}\left\langle b_{i}\right\rangle$, where any $b_{i}$ has order $p$ and let $C=\langle c\rangle$ a group of order $p$. Let $A=B \times C$ and consider, for any $i \in \mathbb{N}$, the automorphism $\gamma_{i}$ of $A$ fixing any element of $B$ and such that $c^{\gamma_{i}}=b_{i} c$. Then $\Gamma=\left\langle\gamma_{i} \mid i \in \mathbb{N}\right\rangle \leq \operatorname{Aut} A$ is power on the $\operatorname{subgroup} B$ of finite index in $A$, but is not $B P$, as $C^{\Gamma}=A$.

(b) Let $A=B \times C$ as in (a) and for any $i \in \mathbb{N}$ consider the automorphism $\delta_{i}$ of $A$ fixing any element of $C$ and any $b_{j}$, if $j \neq i$ and such that 
$b_{i}^{\delta_{i}}=b_{i} c$. The subgroup $\Delta=\left\langle\delta_{i} \mid i \in \mathbb{N}\right\rangle$ of $\operatorname{Aut} A$ is power on the group $A / C$, but is not $A P$, as $B_{\Delta}=1$.

(c) Let $A=A^{\prime} \times A^{\prime \prime}$, with $A^{\prime}=B^{\prime} \times C^{\prime}$ and $\Gamma$ as in (a) (with $B^{\prime}$ and $C^{\prime}$ instead of $B$ and $C$, respectively) and $A^{\prime \prime}=B^{\prime \prime} \times C^{\prime \prime}$ and $\triangle$ as in (b) (with $B^{\prime \prime}$ and $C^{\prime \prime}$ instead of $B$ and $C$, respectively). The group $\Pi=\Gamma \times \Delta \leq \operatorname{Aut} A$ is power on $B^{\prime} A^{\prime \prime} / C^{\prime \prime}$ and hence is $C P$, but is neither AP nor BP.

Proposition 9. Let $\gamma_{1}, \ldots, \gamma_{n}$ automorphisms of an abelian group A. Then

i) if $\gamma_{1}, \ldots, \gamma_{n}$ are $(a p)$, then $\left\langle\gamma_{1}, \ldots, \gamma_{n}\right\rangle \leq \operatorname{Aut} A$ is $(A P)$.

ii) properties $(A P),(B P)$ and $(C P)$ are equivalent for finitely generated groups $\Gamma=\left\langle\gamma_{1}, \ldots, \gamma_{n}\right\rangle$.

Proof. ( $i$ ) In the case $A$ is periodic, we may assume $A$ is a $p$-group and at least one of $\gamma_{i}$ is not elementary. Then $A=D \times R$, where $D=D(A)$ has finite rank and $R$ has finite exponent. For $1 \leq i \leq n$ there exists $E_{i} \leq R$ with finite index on which $\gamma_{i}$ is power. So that each $\gamma_{i}$ is power on $E:=\cap_{i} E_{i}$ and $B:=D E$ has finite index in $A$. By Theorem 2.8 of [3], $\Gamma$ is $(A P)$ on $A$, as wished. For the general case, note that for $1 \leq i \leq n$ there exists $V_{i} \leq A$ which is free abelian such that $\gamma_{i}$ is power on $V_{i}$ and (ap) on the torsion group $A / V_{i}$. So that $\Gamma$ is power on $V:=\cap_{i} V_{i}$ and $(A P)$ on the torsion group $A / V$, by the above. Thus by Theorem 2.10 of [3], $\Gamma$ is $(A P)$ on $A$.

(ii) If $\Gamma$ is $(C P)$, then its elements are $(a p)$ and $(b p)$, by Theorem 1 and Corollary 1 . By the above $\Gamma$ is (AP). It remains to show that if all $\gamma_{i}$ are $(b p)$, then $\Gamma$ is $(B P)$. Again, in the periodic case we may suppose $A$ is a $p$-group and at least one of $\gamma_{i}$ is not elementary, so that $D=D(A)$ has finite rank and $\exp (A / D)=: e<\infty$. Then for $1 \leq i \leq n$ there exists $K_{i}=K_{i}^{\gamma_{i}} \leq A$ such that $K_{i} / D$ is finite and $\gamma_{i}$ is power on $A / K_{i}$. Then $K:=K_{1} \ldots K_{n}$ is $\Gamma$-invariant and $K / D$ is finite. Thus $K[e]$ is finite and, by Theorem 2.11 of [2], $\Gamma$ is (BP) on $A$. In the case $A$ is not periodic, recall that by Theorem 3 for $1 \leq i \leq n$ there exists $V_{i} \leq A$ such that $A / V_{i}$ is torsion and $\gamma_{i}$ is power on $V_{i}$. Then $V:=\cap_{i} V_{i}$ is $\Gamma$-invariant. By the above $\Gamma$ is $(B P)$ on the periodic quotient $A / V$ and by Theorem 2.12 of [2], $\Gamma$ is $(B P)$ on the whole $A$.

THEOREM 4. Let $A$ an abelian non-periodic group $A$ and $\Gamma=$ $\left\langle\gamma_{1}, \ldots, \gamma_{s}\right\rangle \leq$ Aut $A$. Then each $\gamma_{i}$ is inertial iff one of the following holds:

(a) there a finite index subgroup $B$ of $A$ on which each $\gamma_{i}$ acts as a power automorphism.

(b) there are finitely many elements $a_{i} \in A$ and $\gamma \in \Gamma$ such that: 
1. the subgroup $V=\left\langle a_{1}, \ldots, a_{r}\right\rangle^{\langle\gamma\rangle}$ is torsion-free and each $\gamma_{i}$ induces on it a rational-power automorphism $\frac{m_{i}}{n_{i}}\left(m_{i}, n_{i}\right.$ coprime);

2. the factor group $A / V$ is torsion and each $\gamma_{i}$ acts as an almostpower automorphism on it;

3. the $\pi\left(m_{1} n_{1} \cdots m_{s} n_{s}\right)$-component of the torsion subgroup $T$ of $A$ has finite exponent.

Proof. For each $\gamma \in \mathcal{I} \operatorname{Aut}(A)$ denote $\pi(\gamma):=\pi(m n)$ where $\gamma_{\mid A / T}=\frac{m}{n}$.

If all $\gamma_{i}$ 's are $(a p)$, then $\Gamma$ is $(A P)$ by Proposition 9. Thus the statement holds by Theorem 2.10 of [3].

Otherwise, by Theorem 3, $A$ has finite torsion-free rank $r$ and for $1 \leq i \leq s$ there is a torsion-free subgroup $V_{i}$ which is the closure under $\left\langle\gamma_{i}\right\rangle$ of $r$ linearly independent elements such that $\gamma_{i}=\frac{m_{i}}{n_{i}}$ on $V_{i}$ (with $m_{i}, n_{i}$ coprime integers) and $\gamma_{i}$ is (ap) on the periodic factor group $A / V_{i}$. Put $\pi_{i}=\pi\left(m_{i} n_{i}\right)$ and $\pi=\pi\left(m_{1} n_{1} \cdots m_{r} n_{r}\right)$. Then by Theorem 3 the $\pi_{i}$-component of $T$ has finite exponent and hence (3) in the statement holds.

Actually, there is $\gamma \in \Gamma$ such that $\pi(\gamma)=\pi\left(\gamma_{1}\right) \cup \cdots \cup \pi\left(\gamma_{s}\right)=\pi$. This can be seen by induction on $s$, where it is enough to treat the case $s=2$. Let $j=1+\max \left\{i \in \mathbb{N} \mid p^{i}\right.$ divides $m_{1} m_{2} n_{1} n_{2}, p$ prime number $\}$ and put $\gamma=\gamma_{1} \gamma_{2}^{t}$. Then $\gamma=\frac{m_{1} m_{2}^{t}}{n_{1} n_{2}^{t}}$ on $A / T$ and the choice of $t$ ensures $\pi(\gamma)=$ $\pi\left(\gamma_{1}\right) \cup \pi\left(\gamma_{2}\right)$.

Pick Z-independent elements $b_{1}, \ldots, b_{r}$ of $V_{1} \cap \ldots \cap V_{s}$. By Lemma 3 there exists $a_{i} \in\left\langle b_{i}\right\rangle$ such that $\left\langle a_{i}\right\rangle^{\langle\gamma\rangle}$ is torsion-free with rank 1 . Thus we obtain a torsion-free subgroup $V$ of $A$ with shape

$$
V:=\left\langle a_{1}, \ldots, a_{r}\right\rangle^{\langle\gamma\rangle} .
$$

Let us show that $V$ is $\gamma_{i}$-invariant for each $i$. Let $a$ be any element of $V$ and $e=\exp \left(T_{\pi}\right)$. By Theorem $2, V / V_{i}$ is a $\pi$-group and $V$ is $\pi$-divisible. Let $b \in V$ such that $b^{e}=a$. There is a $\pi$-number $u$ such that $b^{u} \in V_{i}$. Since $V_{i}=V_{i}^{\gamma_{i}}$ and $\gamma_{i}=\frac{m_{i}}{n_{i}}$ on $A / T$ we have $b^{\left(n_{i} \gamma_{i}-m_{i}\right) u} \in T \cap V_{i}=1$. It follows $b^{n_{i} \gamma_{i}-m_{i}} \in T_{\pi}$. Therefore $a^{n_{i} \gamma_{i}-m_{i}}=b^{e\left(n_{i} \gamma_{i}-m_{i}\right)}=1$. Thus $V^{\gamma_{i}}=V^{n_{i} \gamma_{i}}=V^{m_{i}}=V$, as claimed. Thus (1) holds, while (2) is trivial as $V$ has maximal torsion-free rank.

Conversely, suppose that conditions (1), (2), (3) hold. By Theorem 3, to show that $\gamma_{i}$ is inertial, we only have to prove that $\gamma_{i}$ is inertial on the periodic group $A / V_{i}$, where $\left.V_{i}=\left\langle a_{1}, \ldots, a_{r}\right\rangle\right\rangle^{\left\langle\gamma_{i}\right\rangle}$. Let $\pi=\pi\left(m_{1} n_{1} \cdots m_{s} n_{s}\right)$ and let $B_{\pi^{\prime}} / V_{i}$ be $\pi^{\prime}$-component of $A / V_{i}$. Then $B_{\pi^{\prime}} / V_{i} \simeq_{\gamma_{i}} B_{\pi^{\prime}} V / V$, which is 
the $\pi^{\prime}$-component of $A / V$ and so $\gamma_{i}$ is inertial on it. Let $p \in \pi$ and let $B_{p} / V_{i}$ be $p$-component of $A / V_{i}$. Since $A / T V$ is a $\pi^{\prime}$-group, $B_{p}$ is contained in $T V$. So $\gamma_{i}$ is power on a subgroup of finite index of $B_{p} / B_{p} \cap V \simeq_{\gamma_{i}} B_{p} V / V$ which has finite exponent. Moreover $\gamma_{i}$ is power on $B_{p} \cap V / V_{i}$, which has finite rank. So by Theorem 2.12 of [2], $\gamma_{i}$ is $(b p)$ and hence inertial on $A / V_{i}$.

\section{REFERENCES}

[1] V. V. Belayev - M. KuzucuoĞLu - E. Seckin, Totally inert groups, Rend. Sem. Mat. Univ. Padova, 102 (1999), pp. 151-156.

[2] C. CASOLO, Groups with finite conjugacy classes of subnormal subgroups, Rend. Sem. Mat. Univ. Padova, 81 (1981), pp. 107-149.

[3] S. Franciosi - F. DE Giovanni - M. L. Newell, Groups whose subnormal subgroups are normal-by-finite, Comm. Alg., 23 (14) (1995), pp. 5483-5497.

[4] L. Fuchs, Infinite Abelian Groups, Academic Press, New York - London, 1970 - 1973.

[5] D. J. S. Robinson, Finiteness conditions and generalized Soluble Groups, Springer V., Berlin, 1972.

[6] D. J. S. Robinson, A Course in the Theory of Groups, Springer V., Berlin, 1982.

[7] D. J. S. Robinson, On inert subgroups of a group, Rend. Sem. Mat. Univ. Padova, 115 (2006), pp. 137-159.

Manoscritto pervenuto in redazione il 12 luglio 2011. 